Letter to the Editor

\title{
Interplay between turbulence, neoclassical and zonal flows during the transition from low to high confinement mode at ASDEX Upgrade
}

\author{
M. Cavedon ${ }^{1,2}$, T. Pütterich ${ }^{1}$, E. Viezzer ${ }^{1}$, G. Birkenmeier ${ }^{1,2}$, T.

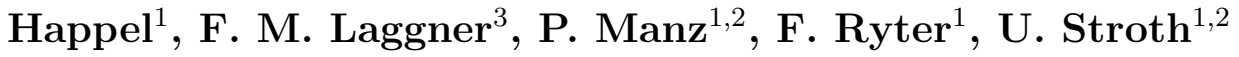 \\ and the ASDEX Upgrade Team
}

E-mail: marco.cavedon@ipp.mpg.de

${ }^{1}$ Max-Plank-Institut für Plasmaphysik, D-85748, Garching, Germany

${ }^{2}$ Physik-Department E28, Technische Universität München, 85748 Garching, Germany

${ }^{3}$ Institute of Applied Physics, TU Wien, Fusion@ÖAW, 1040 Vienna, Austria

March 2016

\begin{abstract}
.
The spatio-temporal interplay between turbulence, mean and zonal flows has been investigated at the L-H and H-L transitions in the edge region of the ASDEX Upgrade tokamak. Close to both transitions, an intermediate phase (I-phase) characterized by "limit cycle like oscillations" (LCOs) is observed in which periodic bursts of turbulence correlate with $\mathbf{E} \times \mathbf{B}$ flow reduction and relaxation of gradients. During the I-phase, the $\mathbf{E} \times \mathbf{B}$ velocity is dominated by the mean flows indicating that turbulence driven flows are small. Periodic dithers between L-mode and the phases with LCOs are also observed just before the $\mathrm{H}$-mode onset where the edge density and temperature profile gradients evolve on similar timescale as the flows. Thus connection between mean and $\mathbf{E} \times \mathbf{B}$ flows holds during the all evolution from L-mode to H-mode demonstrating the fundamental role of the neoclassical flows in the L-H transition physics.
\end{abstract}

In tokamaks, the observed energy and particle losses are in general much larger than the levels predicted by neoclassical theory [1]. In 1982 a regime of improved confinement (H-mode), in which a region of reduced transport is established at the edge of the plasma, was described for the first time [2]. This region is named edge transport barrier (ETB) and is characterized by a strong reduction of turbulence through $\mathbf{E} \times \mathbf{B}$ velocity shear [3, 4].

In toroidally confined plasmas, the $\mathbf{E} \times \mathbf{B}$ velocity is a sum of two contributions: the always present neoclassical or background flows (NFs) and the self induced turbulent flows, also called zonal flows (ZFs). The background flow is dominated by the main ion temperature $\left(T_{\mathrm{i}}\right)$ and density $\left(n_{\mathrm{i}}\right)$ gradients and is roughly equal to the diamagnetic velocity $v_{\mathrm{dia}}^{\mathrm{i}}=\nabla\left(n_{\mathrm{i}} T_{\mathrm{i}}\right) /\left(e Z_{\mathrm{i}} n_{\mathrm{i}}\right)$ with $e$ the elementary charge and $Z_{i}$ the charge number [5]. Zonal flows are azimuthally symmetric, bandlike shear flows 
generated by turbulence stresses [6]. ZFs have been invoked as a possible mechanism for the transition from the highly turbulent low confinement mode (L-mode) to the H-mode often through an intermediate phase (I-phase) which reveals low $\mathrm{kHz}$ oscillations between flows and turbulence. This phase has been observed in many different devices [7, 8, 9, $10,11,12,13,14]$ and was originally called dithering H-mode [14] and more recently IMmode, I-phase or limit cycle oscillations (LCOs) $[15,10,9]$. The existence of zonal flows is often deduced from the phase relation between turbulence amplitude and flows, where a phase difference of $\pi / 2$ is characteristic for predator-prey oscillations $[16,17]$. However, mean field equations also present limit cycle like oscillations as solutions [18]. A direct measurement of the energy transfer from turbulence to the ZF via Reynolds stress, which is not possible in fusion plasmas, has been made in low temperature plasmas [4]. Quantitative comparison between the neoclassical flow and the $\mathbf{E} \times \mathbf{B}$ flow can indicate the strengths of the ZF. Note that a significant level of the Reynolds stress $\Pi_{r}$ cannot be taken as first principle demonstration of zonal flow activity, since any change in $v_{\mathrm{E} \times \mathrm{B}}$, containing also the background flow, may tilt the turbulence eddy orientation and therefore affect $\Pi_{r}[19]$. Moreover, it has been found in simulation that the toroidal coupling of the zonal flow energy to the pressure through the geodesic curvature acts as a loss channel preventing large amplitude, large scale zonal flow from forming [20]. On the other hand, the background flows are emerging as a fundamental player for locking in the transition to H-mode after the initial turbulence suppression [21, 22] and, in certain circumstances, they can alone trigger an ETB [23]. Moreover, there is experimental evidence for the importance of main ions in the $\mathrm{L}-\mathrm{H}$ transition physics [24] and of the edge profile gradients during the LCO $[11,13]$ indicating that the background flow is a fundamental ingredient for the $\mathrm{H}$-mode onset.

In this Letter we make use of an upgraded charge-exchange recombination spectroscopy diagnostic (CXRS, [25]) and Doppler reflectometry [26] to compare directly the spatio-temporal evolution of $\mathbf{E} \times \mathbf{B}$ flows, background flow, and turbulence amplitude during LCOs in the edge region of the ASDEX Upgrade tokamak (AUG). The experimental observations show an intimate connection between the $\mathbf{E} \times \mathbf{B}$ flow and the neoclassically expected flow close to the L-H transition. Every change in $v_{\mathrm{E} \times \mathrm{B}}$ is accompanied by changes in edge gradients demonstrating the fundamental role of the mean flows in the L-H transition physics. During stationary LCOs, the direct comparison between the measured $\mathbf{E} \times \mathbf{B}$ velocity and the ion diamagnetic velocity shows that $v_{\mathrm{E} \times \mathrm{B}}$ is dominated by the main ion gradients at the plasma edge.

The edge charge exchange diagnostic at ASDEX Upgrade provides a complete reconstruction of the impurity profiles and its flows and therefore allows to calculate $E_{r}$ [25]. The active CXRS diagnostic uses light impurity ions $\alpha$ and injected neutral atoms to measure $T_{\alpha}$ and $v_{\alpha}$ from Doppler broadening and shift, and $n_{\alpha}$ from the line intensity. By measuring the poloidal $(\theta)$ and toroidal $(\phi)$ projections of the impurity ion velocity, it is possible to determine the radial electric field $E_{r}$ and hence $v_{\mathrm{E} \times \mathrm{B}}$, with knowledge of $\mathbf{B}$, through the radial force balance equation $E_{r}=\nabla_{r}\left(n_{\alpha} T_{\alpha}\right) /\left(e Z_{\alpha} n_{\alpha}\right)+v_{\phi, \alpha} B_{\theta}-v_{\theta, \alpha} B_{\phi}$. Fifty-two lines of sight (LOS) are focussed within a radial distance of $6 \mathrm{~cm}$ at the low 


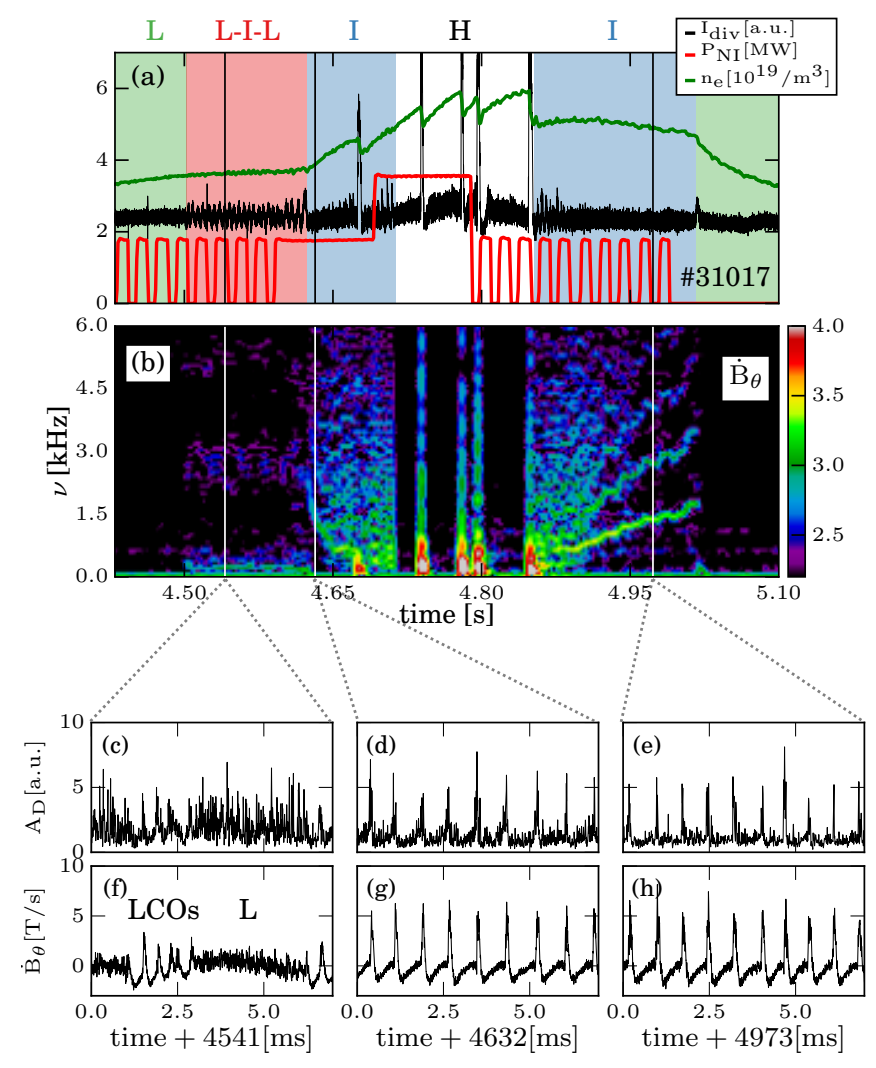

Figure 1: Typical ASDEX Upgrade discharge with NI induced L-H and H-L transition. (a): NI input power $\mathrm{P}_{\mathrm{NI}}$ (red), line integrated edge electron density $n_{\mathrm{e}}$ (green), divertor current $I_{\text {div }}$ (black). (b) spectrogram of $\dot{\mathrm{B}}_{\theta}$ measured at the high field side. (c,d,e)/(f,g,h) turbulence amplitude at the edge by Doppler reflectometry $\mathrm{A}_{\mathrm{D}} / \dot{\mathrm{B}}_{\theta}$ measured during the L-I-L oscillations (c,f) and during the LCO close to the L-H (d,g) and H-L transition $(\mathrm{e}, \mathrm{h})$.

field side plasma edge and have a spatial resolution better than $6 \mathrm{~mm}$ in the pedestal region. Moreover, a new spectrometer allows us to run up to 9 LOS at $20 \mathrm{kHz}$ sampling rate measuring the $\operatorname{He}^{1+}(n=4 \rightarrow 3)$ emission line and therefore permits the study of fast transient phenomenas like the LCO. In order to increase the signal to noise ratio, external helium seeding is indispensable. The $\mathrm{He}^{1+}$ passive emission gives a large contribution of up to $40 \%$ of the total signal at the separatrix. Since the passive signal of $\mathrm{He}^{1+}$ evolves on similar timescales as the active signal, a direct subtraction of the passive component via beam modulation is mandatory to extract localized information. A combination of 4 toroidal and 5 poloidal channels has been selected within $2.5 \mathrm{~cm}$ of the very edge of the confined area in order to diagnose both the $\mathbf{E} \times \mathbf{B}$ velocity and the ion temperature exactly where the ETB is established. Combining these measurements with the electron density $n_{e}$ reconstruction from the lithium beam emission spectroscopy (Li-BES) [27, 28], it is possible to compare the evolution of $v_{\mathrm{E} \times \mathrm{B}}$ and $v_{\text {dia }}^{\mathrm{i}}$ which should be clearly different if ZFs make an important contribution. For this analysis, the impurity 
ion temperature is assumed to be the same as the main ion temperature since the thermal equilibration time between $\mathrm{He}^{2+}$ and $\mathrm{D}^{1+}$ is below $100 \mu \mathrm{s}$ at the plasma edge and dilution of ions by impurity is assumed to be a flat profile, i.e. $\nabla n_{\mathrm{e}} / n_{\mathrm{e}} \approx \nabla n_{\mathrm{i}} / n_{\mathrm{i}}$, which is a good approximation given the typical profiles in L-mode and the purity of the plasmas.

Figure 1 shows the time evolution of a discharge where neutral beam injection (NI) is used to induce an L-H transition. In figure 1(a), the time-traces of the NBI input power (red), the line average electron density from an interferometer channel at the edge (green) and the divertor tile shunt current $I_{\text {div }}$ (black) are shown, while in figure 1 (b) the spectrogram of the poloidal magnetic field $\left(\dot{B}_{\theta}\right)$ measured by a Mirnov-coil located below the inner divertor of AUG is plotted. The divertor tile shunt current is an indicator of heat and particle fluxes reaching the divertor plates and it is often used in metal divertor tokamaks as an L-H transition monitor signal. The discharges analysed in this work are performed at a core electron density of about $4.5 \times 10^{19} \mathrm{~m}^{-3}$, close to the minimum of the power threshold dependence on $n_{e}$, and are carried out using the same heating scheme shown in Figure 1. Two distinct fluctuating phases in the evolution from L-mode to H-mode and back to L-mode are identified: (i) repetitive L-IL transitions (highlighted in red) with a frequency of around $200 \mathrm{~Hz}$, (ii) an extended Iphase limit cycle oscillations at the L-H and H-L transition (highlighted in blue), visible in the spectrogram as harmonics. The L-I-L phase is characterized by a transition from a highly turbulent state (L-mode) to the typical turbulent bursts of the LCOs (fig.1(b)). The turbulence level dynamics at the plasma edge $A_{\mathrm{D}}$, measured by Doppler reflectometry, correlates with the $\dot{\mathrm{B}}_{\theta}$ signal with no delay (fig.1(f)): the spikes of the LCOs are followed by quiet phases while in L-mode a continuously high fluctuation level is found. The same correlation is found during the LCO close to the L-H (figs.1(d) and $1(\mathrm{~g})$ ) and H-L (figs.1(e) and 1(h)) transition. The late I-phase is often interrupted by type-I ELMs $(t=4.675 \mathrm{~s}$, figs.1(a) and $1(\mathrm{~b}))$ indicating that both particle and heat confinement at the edge increase in this phase to a level close to H-mode. This is also confirmed by the signature of the $\mathrm{H}-\mathrm{L}$ transition where the plasma transits back into L-mode only when the LCOs disappear $(t=5.017 \mathrm{~s})$. The dynamic evolution from Lto H-mode described here is preserved in all the discharges analysed later in this work, although differences in the duration and the timing of the different phases changes due to small differences in the electron density at the $\mathrm{L}-\mathrm{H}$ transition and to the intrinsic randomness of the investigated phenomenas. We now focus on the analysis of these two fundamental steps towards the H-mode: the onset of the I-phase and the LCO events.

The L-I-L oscillations give the opportunity to study the transition between L-mode and the LCO in repetitive similar conditions. However, every L-I transition shows a slightly different evolution not allowing conditional averaging and therefore no proper subtraction of the passive CX component can be performed. However, it is possible to compare the relative evolution of the edge gradients $\left(\nabla T_{i}\right.$ and $\left.\nabla n_{e}\right)$ with the edge poloidal $\mathrm{He}^{2+}$ flow, $v_{\theta}^{\mathrm{He}}$, by fitting both the $\mathrm{CX}$ emission of $\mathrm{He}^{2+}$ and the passive $\mathrm{He}^{1+}$ line. This approach is particularly sensitive to changes in the poloidal velocity since, 

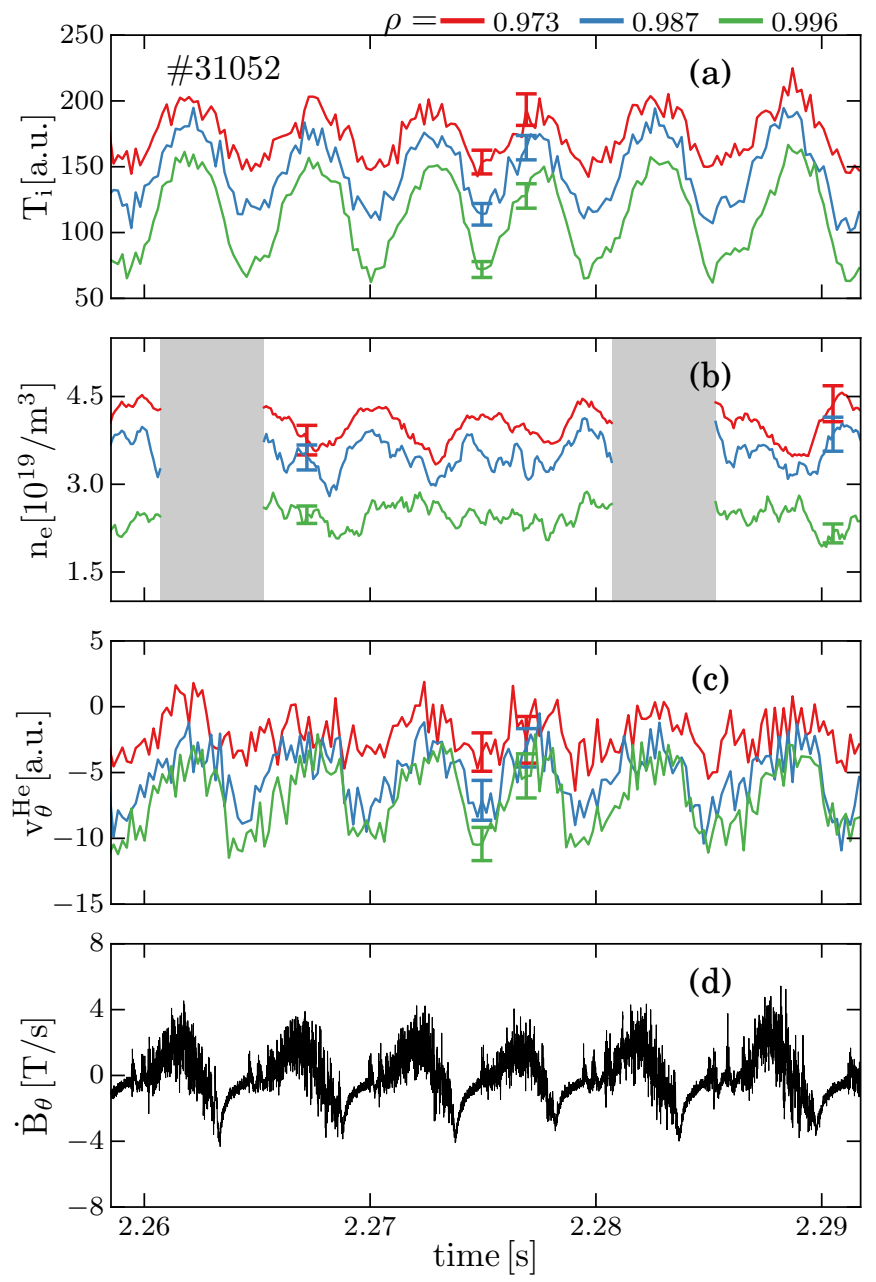

Figure 2: Time-traces during the L-I-L oscillations: signals from different radial positions (a) $T_{i}$, (b) $n_{\mathrm{e}}$, (c) $v_{\theta}^{\mathrm{He}}$, and from (d) $\dot{B}_{\theta}$. The shaded time windows in (b) indicate when the Li-BES is switched off for the background subtraction.

for a certain $E_{r}$, the $\mathrm{He}^{1+}$ ions rotate faster in the poloidal direction compared to the $\mathrm{He}^{2+}$ ions because of their smaller diamagnetic term. Thus, zonal flows could be directly identified if $v_{\theta}^{\mathrm{He}}$ evolves on a different timescale than $\nabla T_{i}$ or $\nabla n_{e}$ or if a delay in the correlation maximum is observed. In the following, the measurements of $T_{i}$ and $v_{\theta}^{\mathrm{He}}$ obtained without accounting for the passive spectra are reported in arbitrary units. Figure 2 shows the time-traces with L-I-L transitions at three different radial positions just inside the separatrix: (a) $T_{i}$, (b) $n_{e}$, (c) $v_{\theta}^{\mathrm{He}}$, (d) $\dot{B}_{\theta}$. The grey shaded area in plot 2 (b) denotes the time interval when the Li-BES is switched off for the quantification of the background emission. No evaluation of the $\mathbf{E} \times \mathbf{B}$ velocity could be obtained from Doppler reflectometry during the I-phase due to the reduced turbulence level between the LCO bursts, resulting in a low signal to noise ratio. A strong modulation of the gradients in $T_{i}$ and $n_{e}$ is observed, coherent with the increase of confinement associated to the LCOs. In particular, the steepening of the edge $\nabla T_{i}$ is established by a reduction of the temperature close to the separatrix while $\nabla n_{e}$ steepens up through the increase of 

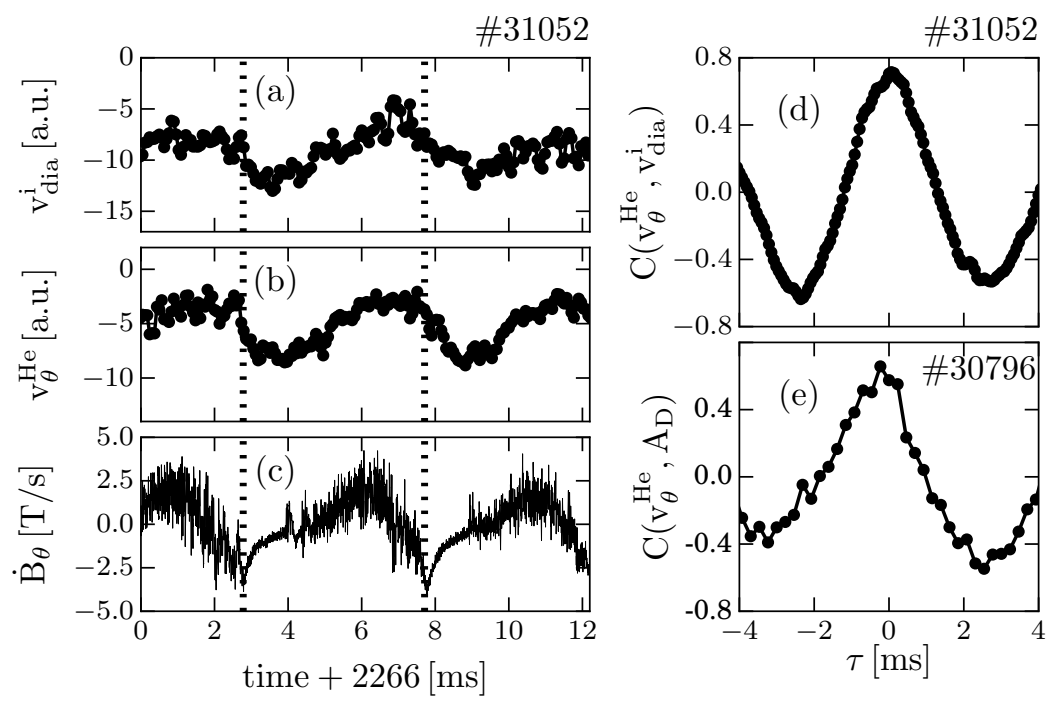

Figure 3: Comparison during the L-I-L dithers between: (a) $v_{\theta}^{i}$, (b) $v_{\theta}^{\mathrm{He}}$ (c) $\dot{B}_{\theta}$ and cross correlation between $v_{\theta}^{i}$ and $v_{\theta}^{\mathrm{He}}(\mathrm{d})$ and between $v_{\theta}^{\mathrm{He}}$ and the turbulence level $A_{D}$.

$n_{e}$ at $\rho \lesssim 0.99$. The evolution of the edge gradients initially extends only $1.0-1.5 \mathrm{~cm}$ just inside the confined region. These substantial changes in the edge $v_{\theta}^{\mathrm{He}}$ and $T_{i}$ profiles are not due to movements of the plasma because its position only oscillates by $1.0-1.5 \mathrm{~mm}$ $(\approx 20 \%$ of the spatial resolution) in between L-mode and the LCOs. The evolution of $v_{\theta}^{\mathrm{He}}$ is compared with $v_{\text {dia }}$, calculated as $\nabla\left(n_{e} T_{i}\right) /\left(n_{e} B\right)$, and $\dot{B}_{\theta}$ during two L-I-L dithers, shown respectively in figures $3(\mathrm{a})-(\mathrm{c})$. A high cross-correlation is found between $v_{\theta}^{\mathrm{He}}$ and $v_{\text {dia }}$ peaked at zero time delay, within the error bars and the experimental resolution (100 $\mathrm{\mu s})$, showing that the edge flows and gradients have similar trends and evolve on the same timescales (fig. $3(\mathrm{~d})$ ). The relative evolution of $v_{\theta}^{\mathrm{He}}$ and of the turbulence level $A_{D}$ from Doppler reflectometry during the L-I-L dithers has been investigated for discharge \#30796 due to the lack of DR data in \#31052. The cross-correlation between $v_{\theta}^{\mathrm{He}}$ and $A_{D}$ is shown in figure $3(\mathrm{e})$ evaluated with a time resolution of $250 \mu \mathrm{s}$. No time delay is observed between the evolution of $v_{\theta}^{\mathrm{He}}$ and $A_{D}$, i.e. $C\left(v_{\theta}^{\mathrm{He}}, A_{D}\right)$ is peaked at zero. However the shape of $C\left(v_{\theta}^{\mathrm{He}}, A_{D}\right)$ is not symmetric and might suggest a delay in the turbulence evolution compared to the flows. This would indicate that the flows, and hence the gradient, develop slightly before the turbulence is suppressed. However, given the time resolution of the measurements and its uncertainties further investigations are necessary to confirm this observation.

A comparison between equilibrium and the total $\mathbf{E} \times \mathbf{B}$ flows during a stationary I-phase is presented in figure 4. Conditional averaging was applied during beam modulation in order to subtract the passive component in the $\mathrm{He}^{2+}$ spectra. In this way it is possible to compare quantitatively the $\mathbf{E} \times \mathbf{B}$ velocity profile (fig. 4(a)) with the diamagnetic velocity of the main ions (fig. 4(b)), measured with a time resolution of $10 \mathrm{kHz}$. Here, the error bars are evaluated from the uncertainties of the impurity flows, the ion temperature and the impurity density gradients using Gaussian error 


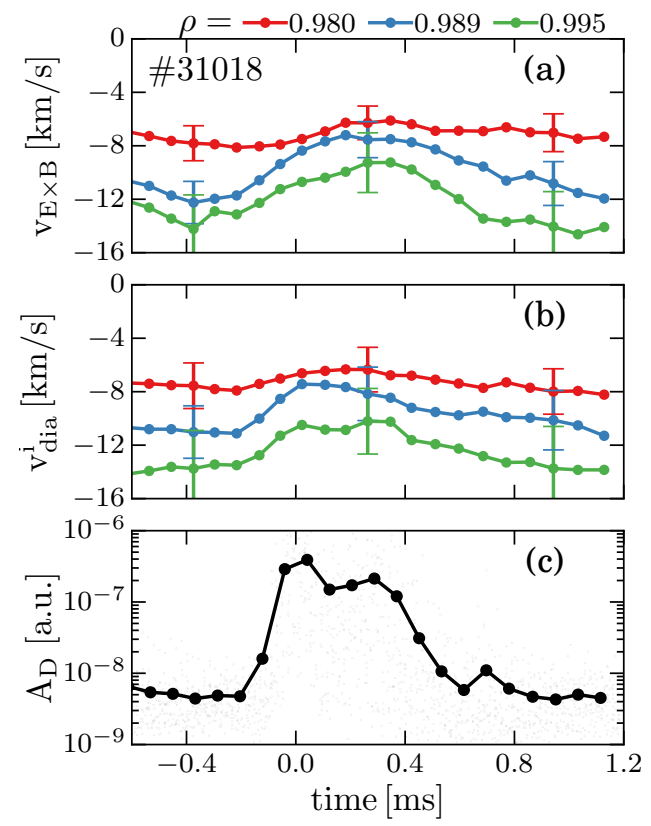

Figure 4: Conditional synchronization of LCO bursts during a stationary I-phase at three different radial positions of: (a) $v_{\mathrm{E} \times \mathrm{B}}$, (b) $v_{\text {dia }}^{\mathrm{i}}$, (c) $\mathrm{A}_{\mathrm{D}}$.

propagation. These are derived in the most conservative approach from the maximum and minimum allowed gradients within the error bars of two neighboring channels. This leads to an overestimation of the error bars which is visible in figure fig. 4(a) and fig. 4(b): the statistical noise of the time traces is much smaller than the error bar itself. The synchronized turbulence amplitude is shown in figure 4(c) where the solid line is the $100 \mu \mathrm{s}$ box average of the points. As previously discussed, the main ion diamagnetic velocity is modulated with the same frequency as the $\mathbf{E} \times \mathbf{B}$ velocity and the edge turbulence. Moreover, in the considered time window, the total shear is dominated by the edge main ion gradients (fig. $5(\mathrm{a})$ ), i.e. $v_{\mathrm{dia}}^{\mathrm{i}} \approx v_{\mathrm{E} \times \mathrm{B}}$, pointing to a physics picture where the ZF amplitude is small compared to the neoclassical flows, in agreement with [11]. Note that this is not only valid during stable LCOs but there are indications that this might extend also to the initial LCO bursts, just after the L-I transition. The maximum of the cross-correlation between $v_{\theta}^{\mathrm{He}}$ and $\nabla\left(n_{e} T_{i}\right)$ calculated in the first $2 \mathrm{~ms}$ after the L-I transition at $t_{0}$, i.e. during the first four LCOs, show good correlation without any time delay within the errorbars confirming that the connection between neoclassical and $\mathbf{E} \times \mathbf{B}$ flows is not broken by ZFs (fig. 5(b)). The $\mathbf{E} \times \mathbf{B}$ flow measured by charge exchange recombination spectroscopy (CXRS), reported in this manuscript, shows an opposite behaviour during the I-phase as compared to the previous Doppler reflectometry measurements [10]. During the I-phase bursts (high turbulence level), the radial electric field, $E_{r}$, well shown in [10] reaches its minimum (indicated as "I high") while in between the bursts (low turbulence level) the $E_{r}$ well is close to zero ("I low"). This behaviour could not be confirmed by means of the CXRS diagnostic which, as reported in figure fig. 4(a), measures the opposite trend: the $E_{r}$ well is deeper when the 

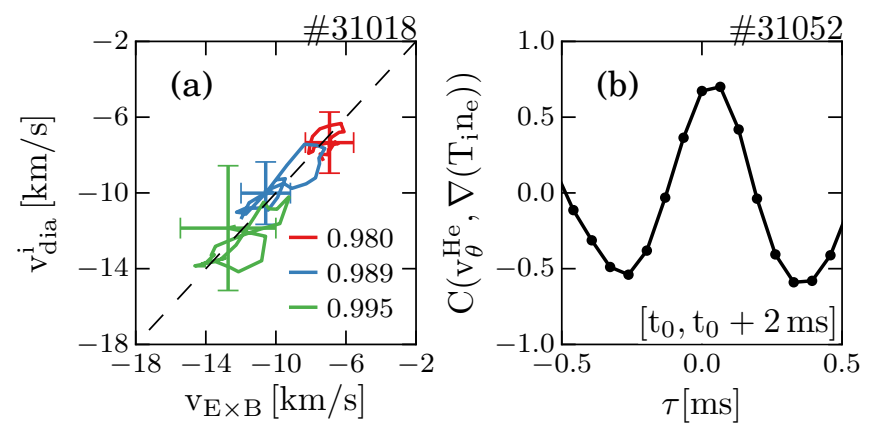

Figure 5: (a) Lissajous diagrams between $v_{\mathrm{E} \times \mathrm{B}}$ and $v_{\mathrm{dia}}^{\mathrm{i}}$ at three different radial position, (b) Cross-correlation between $v_{\theta}^{H e}$ and $\nabla\left(T_{i} n_{e}\right)$ calculated in the first $2 \mathrm{~ms}$ after the L-I transition, i.e. during the first four LCOs.

turbulence is damped while it is reduced when the turbulence level is high. The reason for the disagreement is not fully clear, it might be due to the low signal to noise ratio of the DR in-between the bursts where the reflected signal is low due to the reduced fluctuation level. However, the qualitative trend of the $\mathbf{E} \times \mathbf{B}$ flow during the I-phase reported here is consistent with previous publications [9, 11, 12, 13]. Moreover, in this work particular effort is dedicated to the measurements of the neoclassical flow which combined with the $\mathbf{E} \times \mathbf{B}$ flow, provides the only way to quantitatively estimate the amplitude of the turbulence induced flows. As a result, while the physics of the zonal flows has been largely demonstrated in previous studies [6, and references therein], here it is shown, that in the analysed phases their contribution to the total $\mathbf{E} \times \mathbf{B}$ flow is small compared to the neoclassical one.

To summarize, at AUG the transition from L- to H-mode passes through an Iphase characterized by limit cycle like oscillations. In addition repetitive back an forth transition between L-mode and I-phase are observed. We have provided experimental evidence of the role of the neoclassical flows in the L-H transition physics. Although it has been shown turbulence-zonal flow interaction is present in toroidally confined plasmas, we do not have evidence that it contributes substantially in the phases investigated. Namely, in the dynamic evolution towards the H-mode, the edge profile gradients and $\mathbf{E} \times \mathbf{B}$ flows evolve simultaneously. Moreover, the direct comparison between the main ion diamagnetic velocity and the $\mathbf{E} \times \mathbf{B}$ velocity during a stable Iphase shows that $v_{\mathrm{E} \times \mathrm{B}}$ is dominated by the neoclassical flows.

\section{Acknowledgments}

The authors would like to acknowledge G. D. Conway and P. Hennequin for the valuable discussion and for providing the Doppler reflectometry data. This work has been carried out within the framework of the EUROfusion Consortium and has received funding from the Euratom research and training programme 2014-2018 under grant agreement No 633053. The views and opinions expressed herein do not necessarily reflect those of the European Commission. F.M. Laggner is a fellow of the Friedrich Schiedel Foundation 
for Energy Technology.

\section{References}

[1] J. Hugill. Transport in tokamaks - a review of experiment. Nucl. fusion, 23(3):331, 1983. URL: http://stacks .iop. org/0029-5515/23/i=3/a=006.

[2] F. Wagner et al. Regime of improved confinement and high beta in neutral-beamheated divertor discharges of the asdex tokamak. Phys. rev. lett., 49:1408-1412, 19, November 1982. DOI: 10.1103/PhysRevLett.49.1408. URL: http://link. aps.org/doi/10.1103/PhysRevLett.49.1408.

[3] H. Biglari, P. H. Diamond, and P. W. Terry. Influence of sheared poloidal rotation on edge turbulence. Physics of fluids b: plasma physics, 2(1):1, 1990. ISSN: 08998221. DOI: 10.1063/1 . 859529. URL: http://link. aip.org/link/ PFBPEI/v2/i1/p1/s1\%5C\&Agg=doi.

[4] P. Manz, M. Ramisch, and U. Stroth. Physical mechanism behind zonal-flow generation in drift-wave turbulence. Phys. rev. lett., 103(16):165004, October 2009. ISSN: 0031-9007. DOI: 10.1103/PhysRevLett. 103.165004. URL: http://link. aps.org/doi/10.1103/PhysRevLett.103.165004.

[5] F. L. Hinton and R. D. Hazeltine. Theory of plasma transport in toroidal confinement systems. Rev. mod. phys., 48:239-308, 2, April 1976. DOI: 10.1103/ RevModPhys .48.239. URL: http://link. aps .org/doi/10 .1103/RevModPhys . 48.239 .

[6] P H Diamond, S-I Itoh, K Itoh, and T S Hahm. Zonal flows in plasma - a review. Plasma physics and controlled fusion, 47(5):R35, 2005. URL: http://stacks . iop. org $/ 0741-3335 / 47 / \mathrm{i}=5 / \mathrm{a}=\mathrm{R} 01$.

[7] G. S. Xu et al. First evidence of the role of zonal flows for the $L-H$ transition at marginal input power in the east tokamak. Phys. rev. lett., 107:125001, 12, September 2011. DOI: 10.1103/PhysRevLett.107.125001. URL: http://link. aps.org/doi/10.1103/PhysRevLett.107.125001.

[8] T. Estrada, C. Hidalgo, T. Happel, and P. H. Diamond. Spatiotemporal structure of the interaction between turbulence and flows at the L-H transition in a toroidal plasma. Phys. rev. lett., 107:245004, 24, December 2011. DoI: 10.1103/ PhysRevLett . 107 . 245004. URL: http : / / link . aps . org / doi / 10 . 1103 / PhysRevLett.107.245004.

[9] L. Schmitz et al. Role of zonal flow predator-prey oscillations in triggering the transition to h-mode confinement. Phys. rev. lett., 108(15):155002, April 2012. ISSN: 0031-9007. DOI: 10.1103/PhysRevLett. 108.155002. URL: http://link. aps.org/doi/10.1103/PhysRevLett.108.155002. 
[10] G. D. Conway, C. Angioni, F. Ryter, P. Sauter, and J. Vicente. Mean and oscillating plasma flows and turbulence interactions across the L-H confinement transition. Phys. rev. lett., 106(6):065001, February 2011. ISSN: 0031-9007. DOI: 10.1103/PhysRevLett . 106 .065001. URL: http :// link . aps . org/doi / 10 . 1103/PhysRevLett. 106.065001.

[11] T. Kobayashi et al. Spatiotemporal structures of edge limit-cycle oscillation before L-to-H transition in the jft-2m tokamak. Phys. rev. lett., 111(3):035002, July 2013. ISSN: 0031-9007. DOI: 10.1103/PhysRevLett.111.035002. URL: http://link. aps.org/doi/10.1103/PhysRevLett.111.035002.

[12] S. J. Zweben et al. Quiet periods in edge turbulence preceding the L-H transition in the national spherical torus experiment. Phys. plasmas, 17(10), 102502, 2010. DOI: http://dx.doi .org/10.1063/1.3476276. URL: http://scitation .aip. org/content/aip/journal/pop/17/10/10.1063/1.3476276.

[13] J. Cheng et al. Dynamics of low-intermediate-high-confinement transitions in toroidal plasmas. Phys. rev. lett., 110(26):265002, June 2013. ISSN: 0031-9007. DOI: 10.1103/PhysRevLett.110.265002. URL: http://link .aps.org/doi/10 . 1103/PhysRevLett. 110.265002.

[14] H. Zohm. Dynamic behavior of the L-H transition. Phys. rev. lett., 72:222-225, 2, January 1994. DOI: 10.1103/PhysRevLett.72.222. URL: http://link.aps. org/doi/10.1103/PhysRevLett.72.222.

[15] R. Colchin et al. Slow L-H transitions in DIII-D plasmas. Phys. rev. lett., 88(25):255002, June 2002. ISSN: 0031-9007. DOI: 10 . 1103 / PhysRevLett . 88 . 255002. URL: http://link.aps.org/doi/10.1103/PhysRevLett.88.255002.

[16] P. H. Diamond, Y.-M. Liang, B. A. Carreras, and P. W. Terry. Self-regulating shear flow turbulence: a paradigm for the $L$ to $H$ transition. Phys. rev. lett., 72:2565-2568, 16, April 1994. DOI: 10.1103/PhysRevLett.72.2565. URL: http: //link.aps.org/doi/10.1103/PhysRevLett.72.2565.

[17] Eun-Jin Kim and P. H. Diamond. Zonal flows and transient dynamics of the $L-H$ transition. Phys. rev. lett., 90:185006, 18, May 2003. DOI: 10.1103/PhysRevLett. 90 . 185006. URL: http : // link . aps .org/doi / 10 . 1103/PhysRevLett . 90 . 185006.

[18] Gary M Staebler and R J Groebner. H-mode transitions and limit cycle oscillations from mean field transport equations. Plasma physics and controlled fusion, 57(1):014025, 2015. URL: http : / / stacks . iop . org / 0741-3335/57 / i=1/a= 014025.

[19] Bruce D Scott. Energetics of the interaction between electromagnetic $E \times B$ turbulence and zonal flows. New journal of physics, 7(1):92, 2005. URL: http : //stacks. iop.org/1367-2630/7/i=1/a=092. 
[20] B Scott. The geodesic transfer effect on zonal flows in tokamak edge turbulence. Physics letters a, 320(1):53-62, 2003. ISSN: 0375-9601. DOI: http://dx.doi.org/ 10.1016/j . physleta.2003 .10.080. URL: http: / / www . sciencedirect . com/ science/article/pii/S037596010301644X.

[21] K. Miki et al. Spatio-temporal evolution of the L-I-H transition. Phys. plasmas, 19(9), 092306, 2012. DOI: http://dx.doi.org/10.1063/1.4753931. URL: http: //scitation.aip.org/content/aip/journal/pop/19/9/10.1063/1.4753931.

[22] M. A. Malkov, P. H. Diamond, K. Miki, J. E. Rice, and G. R. Tynan. Linking the micro and macro: L-H transition dynamics and threshold physics. Phys. plasmas, 22(3), 032506, 2015. DOI: http://dx.doi.org/10.1063/1.4914934. URL: http: //scitation.aip.org/content/aip/journal/pop/22/3/10.1063/1.4914934.

[23] K. Miki et al. Physics of stimulated $L \rightarrow H$ transitions. Phys. rev. lett., 110(19):195002, May 2013. DOI: 10.1103/PhysRevLett.110.195002. URL: http: //link.aps.org/doi/10.1103/PhysRevLett.110.195002.

[24] F. Ryter et al. Experimental evidence for the key role of the ion heat channel in the physics of the L-H transition. Nucl. fusion, 54(8):083003, August 2014. ISSN: 0029-5515. DOI: 10 . $1088 / 0029$ - 5515/54/8/083003. URL: http : / / stacks . iop . org / $0029-5515 / 54 / i=8 / a=083003$ ? key = crossref . $41680 \mathrm{f} 22 \mathrm{a} 6 \mathrm{f} 479 \mathrm{~cd} 738 \mathrm{eae} 57 \mathrm{cf} 14 \mathrm{edfd}$.

[25] E. Viezzer et al. High-resolution charge exchange measurements at asdex upgrade. Rev. sci. instrum., 83(10), 103501, 2012. DoI: http://dx.doi.org/10.1063/1. 4755810. URL: http://scitation . aip.org/content/aip/journal/rsi/83/ 10/10.1063/1.4755810.

[26] T. Happel et al. The optimized steerable w-band doppler reflectometer on asdex upgrade: possibilities and issues. In. Proc. $11^{\text {th }}$ International Reflectometry Workshop, Palaiseau, France, 2013.

[27] M Willensdorfer et al. Characterization of the li-bes at asdex upgrade. Plasma physics and controlled fusion, 56(2):025008, 2014. URL: http : / / stacks . iop . org/0741-3335/56/i=2/a=025008.

[28] R Fischer et al. Probabilistic lithium beam data analysis. Plasma physics and controlled fusion, 50(8):085009, 2008. URL: http : / / stacks . iop . org / 0741 $3335 / 50 / i=8 / a=085009$. 